\section{Autorización de uso de emergencia primera vacuna RNA para COVID-19 en Chile}

\author{
MAX ANDRESEN ${ }^{1,2,8}$, RAÚL CORRALES $^{2,3}$, \\ JORGE GALLARDO $^{2,4}$, VIVIANA NORIEGA ${ }^{2,3,5}$, \\ ELIANA SÁNCHEZ ${ }^{2,6}$, CAROLINE WEINSTEIN ${ }^{2,7}$
}

\section{Emergency use authorization for the first RNA vaccine for COVID-19 in Chile}

'Facultad de Medicina Pontificia Universidad Católica de Chile. Santiago, Chile.

IIntegrante Comisión de Evaluación de Productos

Farmacéuticos Nuevos, ANAMED/ISP. Chile.

${ }^{3}$ Clínica Alemana de Santiago y Facultad de Medicina,

Clínica Alemana-Universidad del Desarrollo. Santiago, Chile.

${ }^{4}$ Oncología Médica, Clinica Indisa. Oncología Médica, Universidad de Chile. Santiago, Chile.

${ }^{5}$ Oficina de apoyo a la Investigación Clínica. Hospital Clínico

Universidad de Chile y Facultad de Medicina.

${ }^{6}$ Instituto de Farmacia, Facultad de Ciencias, Universidad

Austral de Chile. Valdivia, Chile.

'Escuela de Química y Farmacia y Centro de Investigación Farmacopea Chilena, Facultad de Farmacia, Universidad de Valparaíso. Valparaíso, Chile.

${ }^{8}$ Editor asociado, Revista Médica de Chile. Chile.

Correspondencia a:

Max Andresen

Revista Médica de Chile.

Bernarda Morín 488. Santiago, Chile.

maxe1991@hotmail.com $\checkmark 1$ 1 Instituto de Salud Pública (ISP) en Chile es el organismo científico técnico encargado del control sanitario de los productos farmacéuticos que se utilizan en el país, garantizando a la población la calidad, seguridad y eficacia de los medicamentos. Es la Autoridad Reguladora Nacional de Referencia Regional y está acreditado con el Nivel IV, máximo estándar del sistema regulatorio Pan Americano. Con fecha 16 de diciembre de 2020 se llevó a cabo la novena sesión-2020 de la Comisión de Evaluación de Productos Farmacéuticos Nuevos del ISP, fecha histórica en la que se otorgó la autorización unánime para el uso de emergencia, no comercial de la primera vacuna, BNT162b2 Pfizer-Biontech, entre otras que podrían llegar a Chile, para enfrentar el avance de la pandemia de COVID-19.

Debido a la pandemia, la comisión de expertos debió sesionar de manera telemática, transmitiéndose vía streaming, en señal de máxima transparencia. Se generó expectación nacional debido a que marcó un hito en la forma de continuar enfrentando la pandemia. Esto se debió en parte a la profusa difusión en los medios de comunicación.

En el mundo se han utilizado diversas plataformas para el desarrollo acelerado de vacunas neutralizantes para el virus SARS-CoV-2. Esta vacuna tiene un nuevo mecanismo, formulado en base al ARN del antígeno viral, primera en su género en recibir autorizaciones de uso de emergencia en otras agencias como MHRA (Reino Unido) y la FDA (EEUU).

Esta autorización de uso en emergencia medicinal, según consta en la Resolución $\mathrm{N}^{\circ} 5155$, del ISP, se ampara en el artículo $\mathrm{N}^{\circ} 99$ del Código Sanitario, no constituye una autorización de Registro Sanitario. Es importante señalar el hecho que las Agencias Regulatorias Internacionales, y también ANAMED, están permitiendo que las fases de los estudios clínicos del desarrollo de las vacunas se realicen en paralelo, de manera de enfrentar este enorme desafío en el menor tiempo posible, esto no significa eliminar etapas o reducir los estándares de seguridad, calidad y eficacia ${ }^{1}$.

El componente activo de esta primera vacuna contra SARS-CoV-2 BNT162b2, presentada a evaluación, es un ARN con nucleótidos modificados que codifica una proteína S de SARS-CoV-2 de longitud completa, encapsulado con nanopartículas lipídicas ${ }^{2}$. La proteína $S$ es vital en la estructura del coronavirus y sirve como proteína de unión, fusión y entrada del virus en las células del huésped, al enlazarse al receptor ACE-2, por el que tiene $10-20$ veces más afinidad que la anterior cepa SARS-CoV ${ }^{3}$.

Debido a la urgencia con la que debimos revisar la documentación, y conscientes de la gran responsabilidad que esta tarea implica, es que como panel de expertos externos optamos por la 
modalidad de trabajar en equipo y tomar de esta manera una decisión de consenso. Para la toma de decisiones y evaluación de la vacuna se tuvo a la vista el Informe Técnico Preliminar del producto, encargado a expertos de la Sociedad de Farmacología de Chile y los antecedentes proporcionados por el laboratorio que incluyeron estudios preclínicos, estudios clínicos de Fase 1 y Fase 2/3 para evaluar la seguridad, tolerabilidad, inmunogenicidad y eficacia, además de las publicaciones en revistas de corriente principal, referentes al desarrollo y grado de avance de las diversas candidatas a vacunas en investigación, teniendo como marco de referencia, los aspectos virológicos y patogénicos de SARS-CoV-2 causante de COVID-19, así como los indicadores epidemiológicos nacionales e internacionales $s^{4,5}$.

\section{Situación actual}

El reporte epidemiológico semanal registra a la fecha 70 millones de casos acumulados y 1,6 millones de fallecidos alrededor del mundo, desde el inicio de la pandemia, con una tasa de letalidad estimada en 4\% (https://www.who.int/ emergencies/diseases/novel-coronavirus-2019/ situation-reports/). En Chile, las cifras oficiales emanadas desde el Ministerio de Salud, señalan que, a la fecha, se ha superado el medio millón de personas contagiadas y reportado cerca de $16 \mathrm{mil}$ fallecidos producto del virus.

Entre los factores biológicos que contribuyen a la mantención del brote, se encuentran los hechos que el virus tiene un periodo de incubación que pueden llegar a 14 días o más, que los pacientes infectados asintomáticos actúen como vectores de su propagación y que los títulos de anticuerpos neutralizantes, en algunos de los convalecientes de COVID-19, declinan rápidamente dejándolos expuestos a una nueva infección ${ }^{6}$.

Lo anterior, asociado a la categórica evidencia de que pacientes adultos mayores, inmunodeprimidos y personas con comorbilidades altamente prevalentes como: hipertensión, diabetes y obesidad tienen mayor mortalidad por COVID-197, ha generado una tragedia, tanto por el número de fallecidos como por la incapacidad de poder controlar la pandemia a nivel mundial. De ahí, la urgente necesidad de contar a la brevedad con vacunas eficaces contra el SARS-CoV-2 que puedan contribuir a frenar su rápida transmisión, poner fin a la condición de pandemia y prevenir eventuales rebrotes.

\section{De la seguridad y eficacia}

A nivel preclínico, la evidencia presentada demostró que BNT162b2 es inmunogénico tanto en ratones, ratas y en primates no humanos, mostrando un perfil de seguridad apropiado en estudios de toxicidad, provocando una respuesta rápida de anticuerpos con títulos neutralizantes medibles de SARS-CoV-2, luego de sólo una dosis y con sustanciales aumentos en los títulos después de una segunda dosis; estos superan los títulos en sueros de los individuos recuperados por SARSCoV-2/COVID-19. Además, se observó una fuerte respuesta celular de linfocitos $\mathrm{T}$ CD4+, CD8+, de interferón $\gamma($ IFN- $\gamma)$, tras la inmunización con BNT162b2, patrón que favorece la eficacia de la vacuna.

Con respecto a los estudios clinicos, los fabricantes presentaron estudios fase 1 y luego fase 2/3 multinacional, doble ciego, controlado con placebo y en curso ${ }^{2}$. Los datos reportados para la fase 1, que evaluó seguridad e inmunogenicidad en adultos jóvenes sanos (mujeres y hombres), mostraron que 2 dosis de $30 \mu \mathrm{g}$ de BNT162b2, separadas en 21 días, generaron altos títulos de anticuerpos neutralizantes contra SARS-CoV-2 y una robusta respuesta celular antígeno-específica de linfocitos T citotóxicos $\mathrm{CD}^{+}$y de linfocitos $\mathrm{T}$ $\mathrm{CD} 4^{+}$tipo $\mathrm{Th} 1^{8}$.

La fase $2 / 3$ fue diseñada para evaluar eficacia y seguridad; se incluyeron 43.548 voluntarios pertenecientes a diferentes grupos raciales $(28,2 \%$ de población hispánica/latina). Los voluntarios recibieron 2 dosis inoculadas vía IM, con 21 días de diferencia, de $30 \mu \mathrm{g}$ de vacuna o placebo. Los pacientes que recibieron BNT162b2 fueron 18.556 y 18.530 recibieron la segunda dosis de placebo, con una aleatorización 1:1. Los datos referentes a seguridad fueron analizados en 2 subgrupos etarios, el primero consideró a los voluntarios desde 16 a 55 años y el segundo subgrupo incluyó a los mayores de 55 años, para analizar reactogenicidad local, eventos sistémicos y uso de medicación tras la administración de la primera y la segunda dosis ${ }^{2}$.

Por otra parte, para establecer la inmunoge- 
nicidad de BNT162b2, se midió el título medio geométrico de IgG neutralizante del SARS-CoV-2. Se detectó un aumento significativo en el día 28, es decir, 7 días después de la segunda dosis, el que se mantuvo alto hasta 1 mes después de la segunda dosis (día 52). Se informó una mediana de seguimiento de eficacia a 2 meses, categorizada en subgrupos, definidos por edad, sexo, raza, etnia, índice de masa corporal y la presencia de condiciones coexistentes, como parte de un seguimiento planificado de 2 años. Este estudio se está llevando a cabo con voluntarios $\geq 12$ años y pacientes $\mathrm{VIH}+$, cuyos resultados no se adjuntaron en la presente solicitud.

La población total incluida en el análisis de eficacia y seguridad fue de 40.137 participantes, de los cuales 36.523 correspondian a participantes sin evidencia previa o existente de infección por SARS-CoV-2. En este grupo se observaron 8 casos de COVID-19, con inicio al menos 7 días después de la segunda dosis, entre los voluntarios tratados con BNT162b2 versus 162 en la rama placebo, lo que corresponde a una eficacia de 95,0\% para la vacuna. Entre los participantes con y sin evidencia de infección previa por SARS CoV-2, se observaron 9 casos de COVID-19 al menos 7 días después de la segunda dosis entre los que recibieron la vacuna y 169 casos entre los que recibieron placebo, lo que corresponde a 94,6\% de eficacia de la vacuna ${ }^{2}$. Se observó un protección temprana que se hizo visible a partir del día 12 después de la administración de la primera dosis de la vacuna.

El seguimiento promedio para aproximadamente la mitad de los participantes fue de 2 meses después de la segunda dosis y 14 semanas para un subgrupo mas reducido. El estudio está en curso y se diseñó para medir eficacia y seguridad con a lo menos en 2 años de seguimiento. Dada la situación de pandemia y la eficacia demostrada para la vacuna es casi imposible hacer el seguimiento previamente establecido para el grupo placebo.

La reactogenicidad sistémica fue más común y severa después de la segunda dosis, que después de la primera, aunque la reactogenicidad local fue similar después las dos dosis y apareció con mayor frecuencia en el subgrupo etario menor.

Los eventos adversos más comunes, no difieren de los efectos reportados para las vacunas contra otras patologías virales, tales como dolor en el sitio de inoculación, fatiga y dolor de cabeza y diarrea, entre los más reportados. Con respecto a eventos adversos graves en los participantes, los inoculados con BNT162b2 se informaron cuatro: lesión en el hombro relacionada con la administración de la vacuna, linfadenopatía axilar derecha, arritmia ventricular paroxística y parestesia en la pierna derecha.

En opinión de este panel, los antecedentes clínicos y preclínicos presentados para el producto BNT162b2 por Pfizer/Biontech, avalan indicación de uso en emergencia para el grupo etario mayor de 16 años, sugiriendo contraindicaciones en personas con antecedentes de hipersensibilidad severa como shock anafiláctico, especialmente a componentes de la vacuna o a otros inyectables (IM, IV, SC), no se cuenta con información aun en sub grupos como: embarazadas o en lactancia, pacientes inmunocomprometidos o con terapia inmunosupresora, pero su empleo se debe definir caso a caso, se debe tener precaución en pacientes en terapia con anticoagulantes o alteraciones de coagulación.

Debe establecerse que no se conoce aún el número de pacientes vacunados que desarrollan la enfermedad asintomática siendo potenciales propagadores de esta, tambien mencionar que el estudio está en curso, por lo que el seguimiento de estos voluntarios vacunados nos entregará el periodo real de inmunización efectiva.

Si bien nuestro panel tiene potestad para pronunciarse en los aspectos relacionados directamente con seguridad y eficacia, el hecho que esta vacuna deba almacenarse a ultra baja temperatura $\left(-60\right.$ a $\left.-80^{\circ} \mathrm{C}\right)$, para evitar su degradación, hace necesario que se advierta que además de la estrecha farmacovigilancia indicada, se elaboren estrictos protocolos de seguimiento de la preservación de la cadena de frío.

\section{Integrantes Comisión de expertos integrantes de la Evaluación de Productos Farmacéuticos Nuevos. ANAMED/ISP}

Max Andresen

Facultad de Medicina Pontificia Universidad Católica de Chile.

\section{Raúl Corrales}

Clínica Alemana de Santiago y Facultad de Medicina, Clínica Alemana-Universidad del Desarrollo. 


\section{Jorge Gallardo}

Oncología Médica, Clínica Indisa. Oncología Médica, Universidad de Chile.

\section{Viviana Noriega ${ }^{\star}$}

Oficina de apoyo a la Investigación Clínica. Hospital Clínico Universidad de Chile y Facultad de Medicina.

\section{Eliana Sánchez}

Instituto de Farmacia, Facultad de Ciencias, Universidad Austral de Chile.

\section{Caroline Weinstein}

Escuela de Química y Farmacia y Centro de Investigación Farmacopea Chilena, Facultad de Farmacia, Universidad de Valparaíso.

${ }^{*}$ No participó en la comisión para la evaluación de la vacuna por declarar conflicto de interés.

\section{Referencias}

1. Hodgson SH, Mansatta K, Mallett G, Harris V, Emary KR, Pollard AJ. What defines an efficacious COVID-19 vaccine? A review of the challenges assessing the clinical efficacy of vaccines against SARS-CoV-2. The Lancet
Infectious Diseases 2020. https://doi.org/10.1016/S14733099(20)30773-8.

2. Polack FP, Thomas SJ, Kitchin N, Absalon J, Gurtman A, Lockhart S, et al. Safety and efficacy of the BNT162b2 mRNA covid-19 vaccine. N Engl J Med 2020. DOI: 10.1056/NEJMoa2034577.

3. Samrat SK, Tharappel AM, Li Z, Li H. Prospect of SARS-CoV-2 spike protein: Potential role in vaccine and therapeutic development. Virus Res 2020; 198141. https://doi.org/10.1016/j.virusres.2020.198141.

4. Poland GA, Ovsyannikova IG, Kennedy RB. SARSCoV-2 immunity: review and applications to phase 3 vaccine candidates. Lancet 2020; 396: 1595-606.

5. Mahase E. Vaccinating the UK: how the COVID vaccine was approved, and other questions answered. BMJ 2020; (Online): 371.

6. Long Q-X, Tang X-J, Shi Q-L, Li Q, Deng H-J, Yuan J, et al. Clinical and immunological assessment of asymptomatic SARS-CoV-2 infections. Nat Med 2020; 26 : 1200-4. https://doi.org/10.1038/s41591-020-0965-6.

7. Zhou F, Yu T, Du R, Fan G, Liu Y, Liu Z, et al. Clinical course and risk factors for mortality of adult inpatients with COVID-19 in Wuhan, China: a retrospective cohort study. Lancet 2020; 395: 1054-62.

8. Walsh EE, Frenck Jr RW, Falsey AR, Kitchin N, Absalon J, Gurtman A, et al. Safety and immunogenicity of two RNA-based Covid-19 vaccine candidates. N Engl J Med 2020. DOI: 10.1056/NEJMoa2027906. 